\title{
Solving the Christoffel equation: phase and group velocities
}

\author{
Jan W. Jaeken ${ }^{\mathrm{a}, *}$, Stefaan Cottenier ${ }^{\mathrm{a}, \mathrm{b}}$ \\ ${ }^{a}$ Center for Molecular Modeling, Ghent University, Technologiepark 903, 9052 \\ Zwijnaarde, Belgium \\ ${ }^{b}$ Department of Material Science and Engineering, Technologiepark 903, 9052 \\ Zwijnaarde, Belgium
}

\begin{abstract}
We provide christoffel, a Python tool for calculating direction-dependent phase velocities, polarization vectors, group velocities, power flow angles and enhancement factors based on the stiffness tensor of a solid. It is built in a modular way to allow for efficient and flexible calculations, and the freedom to select and combine results as desired. All derivatives are calculated analytically, which circumvents possible numerical sampling problems. GNUPlot scripts are provided for convenient visualization.
\end{abstract}

Keywords: Christoffel equation; Acoustic waves; Sound velocity; Phase velocity; Group velocity; Enhancement factor

\section{PROGRAM SUMMARY}

Program Title: Christoffel

Journal Reference:

Catalogue identifier:

Licensing provisions: GNU General Public Licence, version 3

Programming language: Python

Computer: Workstations

Operating system: Linux/UNIX/Windows/MacOS

Keywords: Christoffel equation, Stiffness tensor, Acoustic waves, Sound velocity, Phase velocity, Group velocity, Enhancement factor

Classification: 7.8

\footnotetext{
${ }^{*}$ Corresponding author.

E-mail address: jan.jaeken@ugent.be
} 
External routines/libraries: NumPy

Nature of problem: Calculating acoustic phase and group velocities in homogeneous solids

Solution method: Solving the Christoffel equation eigenvalue problem by diagonalization; calculating group velocities and enhancement factors analytically as derivatives.

Running time: Seconds/minutes

\section{Introduction}

An important relation in material science is the connection between the stiffness tensor and sound velocities of a solid. The stiffness tensor of a material characterizes its behaviour under stress in the elastic regime. It is represented either by a 4-tensor in 3D space with 81 components (of which 21 are independent), or as a symmetric $6 \times 6$ matrix. The 21 independent matrix elements of the latter matrix are called the elastic constants, quantifying the material's resistance to specific strains. Since sound is a form of elastic waves travelling in a medium, the stiffness tensor will contain information about how these sound waves behave. Conversely, experimental determination of sound velocities is a common way to determine elastic constants[1].

Knowing the elastic constants allows to predict the sound velocities in a material by solving the Christoffel equation. Such independent knowledge on the elastic constants can be obtained, for instance, by ab initio calculations[2] and has recently become a routine activity[3].

For the calculation of phase and group velocities and the enhancement factor from elastic constants using the Christoffel equation, currently only one fully open-source tool is available: AWESoMe[4], which is written in Fortran90. We wish to provide a Python code which is flexible, reliable, importable as a module and easily customizable. Simultaneously, we want to rely as little as possible on the numerical determination of derivatives to ensure reliable results, even for highly anisotropic materials.

This paper is structured as follows: Section 2 gives the theoretical background on quantities related to velocities of non-dispersive elastic waves in solids. Section 3 expands upon practical ways to calculate these quantities analytically using standard linear algebra techniques. In section 4 the working and use of the christoffel module is explained, while section 5 details 
the visualization of the output generated. Finally, a summary is given and an outlook is sketched in section 6 .

\section{Theory}

\subsection{Christoffel equation}

The stiffness tensor $\mathbf{C}$ is a fundamental property of a material. It generalizes Hooke's law in three dimensions, relating strains and stresses in the elastic regime.

$$
\sigma_{i j}=\sum_{n m} C_{i j n m} \epsilon_{n m}
$$

where $\sigma$ is the stress tensor and $\epsilon$ is the strain tensor. The sum, as every sum in this paper, runs over the three Cartesian coordinates. Often the stiffness tensor is written as a $6 \times 6$ matrix

$$
\left(\begin{array}{c}
\sigma_{1} \\
\sigma_{2} \\
\sigma_{3} \\
\hdashline \sigma_{4} \\
\sigma_{5} \\
\sigma_{6}
\end{array}\right)=\left(\begin{array}{ccc:ccc}
C_{11} & C_{12} & C_{13} & C_{14} & C_{15} & C_{16} \\
C_{12} & C_{22} & C_{23} & C_{24} & C_{25} & C_{26} \\
C_{13} & C_{23} & C_{33} & C_{34} & C_{35} & C_{36} \\
\hdashline C_{14} & C_{24} & \bar{C}_{34} & C_{44} & C_{45} & C_{46}^{-} \\
C_{15} & C_{25} & C_{35} & C_{45} & C_{55} & C_{56} \\
C_{16} & C_{26} & C_{36} & C_{46} & C_{56} & C_{66}
\end{array}\right)\left(\begin{array}{c}
\epsilon_{1} \\
\epsilon_{2} \\
\epsilon_{3} \\
\hdashline 2 \epsilon_{4} \\
2 \epsilon_{5} \\
2 \epsilon_{6}
\end{array}\right)
$$

through the use of Voigt notation, defined as

$$
\begin{array}{l:l}
1 \rightarrow x x & 4 \rightarrow y z \\
2 \rightarrow y y & 5 \rightarrow x z \\
3 \rightarrow z z & 6 \rightarrow x y .
\end{array}
$$

The factors of 2 in the strain tensor are to ensure (2) corresponds to (1) by taking proper care of double counting.

The stiffness tensor not only contains information about static deformations, but also about elastic waves traveling through the material. Determining the dispersion relation for these waves is possible by solving the Christoffel equation [5].

$$
\sum_{i j}\left[M_{i j}-\rho \omega^{2} \delta_{i j}\right] s_{j}=0
$$

for a monochromatic plane wave with wave vector $\mathbf{q}$, frequency $\omega$ and polarization $\hat{\mathbf{s}}$ in a material with density $\rho . \mathbf{M}$ is the Christoffel matrix, defined as

$$
M_{i j}=\sum_{n m} q_{n} C_{i n m j} q_{m}
$$


This is a simple eigenvalue problem that can be routinely solved for arbitrary q. The result is a set of three frequencies and polarizations for each value of $\mathbf{q}$. Since $\mathbf{M}$ is real and symmetric, the eigenvalues are real and vectors $\left\{\hat{\mathbf{s}}_{n}\right\}$ constitute an orthogonal basis. Furthermore, the requirement that $\mathbf{C}$ be positive definite ensures that $\omega^{2}$ is not only real but strictly positive.

To reduce notational clutter, we will use the reduced stiffness tensor $\mathbf{C}^{\prime}=\mathbf{C} / \rho$ and the corresponding reduced Christoffel matrix. For further simplification, consider that in a single direction, $\omega(\mathbf{q})$ is a linear function of $q$. This is easily seen by inspecting (4) and (5). The sound velocities are clearly independent of wavelength. In a one-dimensional case, this would mean there are only three possible solutions which are independent of $q$. However, the solutions of the Christoffel equation are very much dependent upon the direction of $\mathbf{q}$.

Due to the wavelength-independence of the velocities, we consider $\mathbf{q}$ to not have dimension of inverse length, but to be a dimensionless unit vector denoting only the direction of travel of a monochromatic plane wave. This changes the dimension of the reduced Christoffel matrix from frequency squared to that of velocity squared. Combining all this reduces (4) to

$$
\sum_{i j}\left[M_{i j}-v_{p}^{2} \delta_{i j}\right] s_{j}=0,
$$

where $v_{p}$ is the velocity of a monochromatic plane wave traveling in the direction of $\hat{\mathbf{q}}$. The prime on the reduced Christoffel matrix has been dropped for convenience.

The determination of a material's sound velocities based on the (reduced) stiffness tensor is a straightforward eigenvalue problem. From (6) we obtain three velocities, one primary $(\mathrm{P})$ and two secondary $(\mathrm{S})$, which correspond to the (pseudo-)longitudinal and (pseudo-)transversal polarizations, respectively. The eigenvectors provide the (unsigned) polarization directions.

\subsection{Phase, group, power flow, enhancement}

Up until now, only the velocity of a monochromatic plane wave has been considered. This is generally refered to as the phase velocity. However, real sound is never purely monochromatic, nor purely planar. Let us consider a wave packet with a small spread in wavelength and direction of travel. The velocity of the wave packet formed by the superposition of these phase waves is called the group velocity. It is the velocity at which acoustic energy travels 
through a homogeneous, non-dispersive medium[5], and is given by

$$
\mathbf{v}_{g} \equiv \vec{\nabla} v_{p}
$$

The gradient is taken in reciprocal space (which we consider to be dimensionless), and is a derivative to the components of $\hat{\mathbf{q}}$.

Note that $v_{p}$ is a scalar function of $\hat{\mathbf{q}}$, while $\mathbf{v}_{g}$ is a vector-valued function, which generally does not lie in the direction of $\hat{\mathbf{q}}$. This angle between phase and group velocity is called the power flow angle $\psi$ and is defined as

$$
v_{p}=v_{g} \cos (\psi)
$$

Equivalently,

$$
\cos (\psi)=\hat{\mathbf{n}}_{p} \cdot \hat{\mathbf{n}}_{g}
$$

where $\hat{\mathbf{n}}_{p}$ and $\hat{\mathbf{n}}_{g}$ are the normalized directions of the phase and group velocities respectively.

The group velocity direction is the direction in which energy travels. As this generally does not correspond to the phase velocity direction, there will be variations in power flow concentration as a function of direction (see figure 1)). This effect is quantified by the enhancement factor [6]

$$
A=\frac{\Delta \Omega_{p}}{\Delta \Omega_{g}}
$$

where $\Delta \Omega_{p}$ is the solid angle spanned by a set of phase wave vectors $\hat{\mathbf{n}}_{p}$ and $\Delta \Omega_{g}$ is the solid angle spanned by the equivalent set of group wave vectors $\hat{\mathbf{n}}_{g}$. When using normalized vectors, this corresponds to the area of the tetragon they span on the unit sphere (see figure 2).

\subsection{The enhancement factor: infinitesimal}

Infinitesimally, the enhancement factor is most easily considered in spherical coordinates. The solid angle equals the area of the infinitesimal paralellogram on the unit sphere spanned by the (vector-valued) partial derivatives of the phase or group vectors to $\theta$ and $\phi$. The area is then given by the absolute value of the vector product.

$$
d \Omega_{p}=\sin (\theta) d \theta d \phi
$$

and

$$
d \Omega_{g}=\left\|\frac{\partial \hat{\mathbf{n}}_{g}}{\partial \theta} \times \frac{\partial \hat{\mathbf{n}}_{g}}{\partial \phi}\right\| \sin (\theta) d \theta d \phi
$$




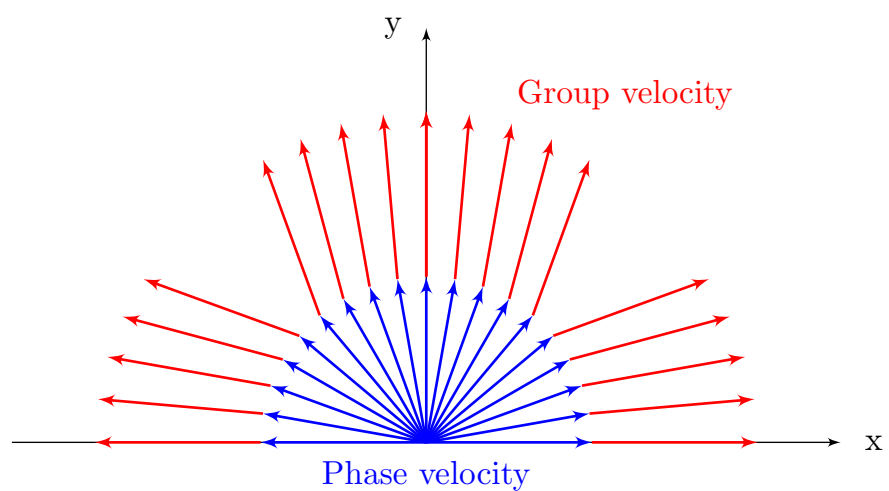

Figure 1: A cartoon to illustrate how phase and group vectors can differ in direction. In this example, the acoustic energy is focused towards the $\mathrm{x}$ and $\mathrm{y}$ directions.
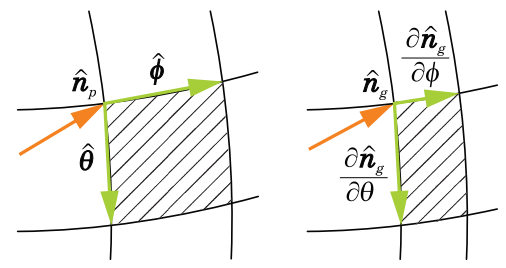

Figure 2: $d \Omega_{p}$ and $d \Omega_{g}$

from which the enhancement factor follows as

$$
A=\left\|\frac{\partial \hat{\mathbf{n}}_{g}}{\partial \theta} \times \frac{\partial \hat{\mathbf{n}}_{g}}{\partial \phi}\right\|^{-1} .
$$

The vector product in equation (12) can be rewritten as

$$
\left(\hat{\boldsymbol{\theta}} \cdot \vec{\nabla} \hat{\mathbf{n}}_{g}\right) \times\left(\hat{\boldsymbol{\phi}} \cdot \vec{\nabla} \hat{\mathbf{n}}_{g}\right)=\operatorname{Cof}\left(\vec{\nabla} \hat{\mathbf{n}}_{g}\right) \cdot(\hat{\boldsymbol{\theta}} \times \hat{\boldsymbol{\phi}})=\operatorname{Cof}\left(\vec{\nabla} \hat{\mathbf{n}}_{g}\right) \cdot \hat{\mathbf{q}}
$$

where $\hat{\boldsymbol{\theta}}=\frac{\partial \hat{\mathbf{q}}}{\partial \theta}$ and $\hat{\boldsymbol{\phi}}=\frac{\partial \hat{\mathbf{q}}}{\partial \phi}$. $\operatorname{Cof}()$ signifies the matrix of cofactors $\operatorname{Cof}(\mathbf{X})=$ $\operatorname{det}(\mathbf{X}) \mathbf{X}^{-T}$. The enhancement factor is then

$$
A=\left\|\operatorname{Cof}\left(\vec{\nabla} \hat{\mathbf{n}}_{g}\right) \cdot \hat{\mathbf{q}}\right\|^{-1}
$$

The latter expression no longer relies on spherical coordinates, avoiding any difficulties due to poorly defined derivates at the poles. Furthermore, it is directly calculable from derivatives of the eigenvalues of the Christoffel matrix. This differs from, but is equivalent to, the expression used by Northrop et al[7], who focused on computational efficiency over flexibility. 


\section{Implementation}

A strategy for an accurate determination of the aforementioned properties is sketched in figure 3. By using the gradient and Hessian of the Christoffel matrix, one can obtain exact results for the group velocity and enhancement factor.

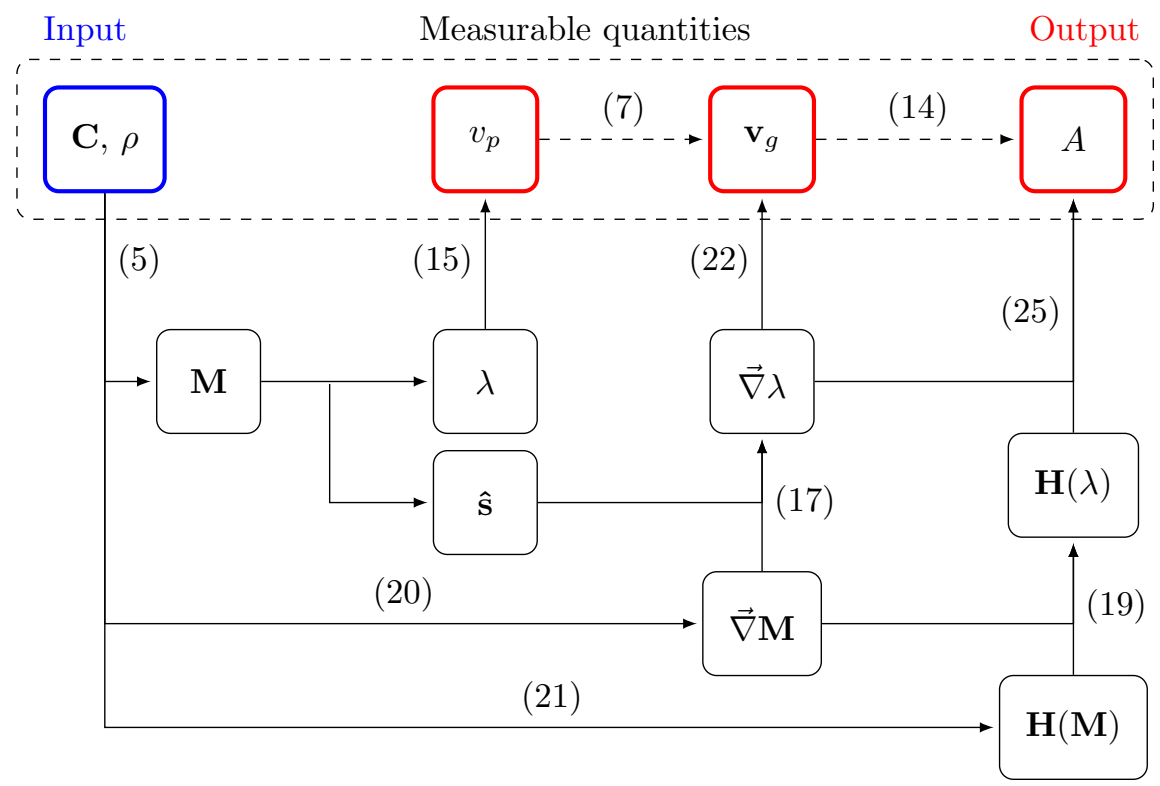

Figure 3: Flowchart showing how quantities are calculated from one another. The input consists of the stiffness tensor and density of the material. To determine phase velocities, group velocities and the enhancement factor, one must calculate the eigenvalues of the Christoffel matrix and their derivatives. Full lines indicate calculations while dashed lines are definitions. The numbers refer to the corresponding equations in this text.

The practical strategy relies on recasting the equations of section 2 as functions of (the derivatives of) the eigenvalues of the Christoffel matrix. For example, the eigenvalues of the Christoffel matrix are the square of the phase velocity:

$$
\lambda=v_{p}^{2}
$$

from which it readily follows that

$$
\mathbf{v}_{g}=\vec{\nabla} v_{p}=\vec{\nabla} \sqrt{\lambda}=\frac{\vec{\nabla} \lambda}{2 \sqrt{\lambda}} .
$$




\subsection{Derivatives of eigenvalues and eigenvectors}

To calculate the aforementioned properties analytically, we need expressions for the first and second derivatives of the eigenvalues of a matrix, as function of the derivatives of the matrix itself. Generally, the expression for the first derivative of an eigenvalue $\lambda$ of a matrix $M$ is given by:

$$
\frac{\partial \lambda_{i}}{\partial q_{k}}=\hat{\mathbf{s}}_{i} \cdot \frac{\partial \mathbf{M}}{\partial q_{k}} \cdot \hat{\mathbf{s}}_{i}
$$

where $\hat{\mathbf{s}}_{i}$ is the normalized eigenvector corresponding to eigenvalue $\lambda_{i}$. Higher order derivatives require an expression for the derivatives of the eigenvectors as well. These are given by[8]

$$
\frac{\partial \hat{\mathbf{s}}_{i}}{\partial q_{k}}=\left(\lambda_{i} \mathbf{I}-\mathbf{M}\right)^{+} \cdot \frac{\partial \mathbf{M}}{\partial q_{k}} \cdot \hat{\mathbf{s}}_{i}
$$

where ()$^{+}$denotes the pseudo-inverse. Using the above, the second order derivative of an eigenvalue equals

$$
\frac{\partial^{2} \lambda_{i}}{\partial q_{k} \partial q_{m}}=\hat{\mathbf{s}}_{i} \cdot \frac{\partial^{2} \mathbf{M}}{\partial q_{k} \partial q_{m}} \cdot \hat{\mathbf{s}}_{i}+2 \hat{\mathbf{s}}_{i} \cdot \frac{\partial \mathbf{M}}{\partial q_{k}} \cdot\left(\lambda_{i} \mathbf{I}-\mathbf{M}\right)^{+} \cdot \frac{\partial \mathbf{M}}{\partial q_{m}} \cdot \hat{\mathbf{s}}_{i} .
$$

\subsection{Derivatives of the Christoffel matrix}

Due to the simple form of the Christoffel matrix (5), the rank 3 tensor $\vec{\nabla} \mathbf{M}$ is easily determined to be

$$
\frac{\partial M_{i j}}{\partial q_{k}}=\sum_{m}\left(C_{i k m j}+C_{i m k j}\right) q_{m}
$$

which is a function of $\hat{\mathbf{q}}$. Note that (20) and (17) make the calculation of group velocities trivial once the eigenvalues and eigenvectors of the Christoffel matrix have been determined.

The Hessian of the Christoffel matrix is independent of $\hat{\mathbf{q}}$ and given by

$$
\frac{\partial^{2} M_{i j}}{\partial q_{k} \partial q_{m}}=C_{i k m j}+C_{i m k j}
$$

Note that (21) has no implicit summation over indices, each matrix element is given by the sum of just two tensor components. These two terms are generally not equal. 


\subsection{Determining the group velocity and enhancement factor}

The $i$-th group velocity is calculated by combining (16), (17) and (20):

$$
\mathbf{v}_{g}^{i}=\frac{\hat{\mathbf{s}}_{i} \cdot \vec{\nabla} \mathbf{M} \cdot \hat{\mathbf{s}}_{i}}{2 \sqrt{\lambda_{i}}} .
$$

The implementation of the enhancement factor is slightly more involved, as it deals with the derivatives of normalized vectors. For a general differentiable, strictly positive vector field $\mathbf{v}$, the gradient of the normalized field equals

$$
\vec{\nabla} \frac{\mathbf{v}}{\|\mathbf{v}\|}=\frac{\vec{\nabla} \mathbf{v}}{\|\mathbf{v}\|}-\frac{\mathbf{v} \otimes(\vec{\nabla} \mathbf{v}) \cdot \mathbf{v}}{\|\mathbf{v}\|^{3}}
$$

where $\otimes$ denotes the outer product $(\mathbf{a} \otimes \mathbf{b})_{i j}=a_{i} b_{j}$. Note that $(23)$ is an expression for the gradient of the vector field, not the divergence. The gradient of a vector is a rank 2 tensor, while the divergence yields a scalar.

The gradient of the normalized group velocity as a function of the eigenvalues of the Christoffel matrix is given by

$$
\vec{\nabla} \hat{\mathbf{n}}_{g}=\vec{\nabla} \frac{\mathbf{v}_{g}}{\left\|\mathbf{v}_{g}\right\|}=\vec{\nabla} \frac{\vec{\nabla} v_{p}}{\left\|\vec{\nabla} v_{p}\right\|}=\vec{\nabla} \frac{\vec{\nabla} \lambda}{\|\vec{\nabla} \lambda\|}
$$

which combined with (23) yields

$$
\vec{\nabla} \hat{\mathbf{n}}_{g}=\frac{\mathbf{H}(\lambda)}{\|\vec{\nabla} \lambda\|}-\frac{\vec{\nabla} \lambda \otimes \mathbf{H}(\lambda) \cdot \vec{\nabla} \lambda}{\|\vec{\nabla} \lambda\|^{3}}
$$

where $\mathbf{H}$ is the Hessian operator. Although (25) looks like it would yield a symmetric matrix, note that the outer product does not commute. This means that in general, $\vec{\nabla} \hat{\mathbf{n}}_{g} \neq\left(\vec{\nabla} \hat{\mathbf{n}}_{g}\right)^{T}$.

Once $\vec{\nabla} \lambda$ is calculated using (17) and $\vec{\nabla} \hat{\mathbf{n}}_{g}$ using (25), (14) provides the enhancement factor.

\section{The Christoffel module}

The full mathematical machinery as described in section 3 is contained in the christoffel python module. The central object is the Christoffel object, which contains all functions needed to calculate $v_{p}, \mathbf{v}_{g}, \psi$ and $A$ from 
the (reduced) stiffness tensor and (normalized) wave vector. Upon initialization (requiring a stiffness tensor and density), all direction-independent data is calculated once and stored. To find direction-dependent properties, one sets a wave vector (using spherical or cartesian coordinates). Everything is calculated on a need-to-know basis to prevent unnecessary computational load. For example, using the get_group_velocity() function will cause phase and group velocities to be calculated if they haven't already, but not the enhancement factor. An extra routine is also available to allow users to calculate the enhancement factor via a numerical approach, for when the analytical derivative is ill-defined.

A second script named scansurface is provided which uses the christoffel module to scan the $z \geq 0$ half of the unit sphere. A settings file allows for the input of the stiffness tensor and density, and to choose the sampling density of angular coordinates $\theta$ (polar) and $\phi$ (azimutal). It is possible to provide a rotation matrix to rotate the stiffness tensor, which can be useful when comparing output from different crystal structures. Furthermore, Gnuplot scripts are available to conveniently plot the data generated in this way. This is not the only possible implementation however, the stand-alone module allows for flexibility and versatility such that the user can choose which functionality they need, and build upon the tools that are provided.

\section{Output and visualization}

The data is stored in 3 files, one for each mode named slow_secondary.dat, fast_secondary.dat and primary.dat. Each file contains the following for each direction in the grid:

- The spherical coordinates $\theta$ and $\phi$

- The projection of $\hat{\mathbf{q}}$ onto the unit cube

- The phase velocity $v_{p}$ in $\mathrm{km} / \mathrm{s}$

- The phase velocity $v_{p}$ relative to the corresponding isotropic sound velocity

- Polarization direction of the sound wave

- The group velocity $v_{g}$ in $\mathrm{km} / \mathrm{s}$ 
- The group velocity $v_{g}$ relative to the corresponding isotropic sound velocity

- $x, y, z$-coordinates of the ray surface in $\mathrm{km} / \mathrm{s}$

- The power flow angle $\psi$ in degrees

- The enhancement factor, $A$

GNUPlot scripts are provided for convenient plotting of these quantities, as shown in figures 4 through 9 for the example of GaAs. Many different plotting options are available with color palettes chosen in an attempt to represent the data in the most faithful way. For all properties, there is the possibility to project the data on the unit sphere and the unit cube as shown in figure 4. There are also two 2-dimensional representations: the Lambert equalarea projection (which preserves local area) and the stereographic projection (which preserves local shape) as shown in figure 5. Furthermore, the phase and group sound velocities can be represented either in absolute units, or relative to the isotropic sound velocities (see figure 4). These isotropic sound velocities are given by

$$
\begin{gathered}
v_{\text {iso }}^{P}=\sqrt{\frac{B+\frac{4}{3} G}{\rho}} \\
v_{\text {iso }}^{S}=\sqrt{\frac{G}{\rho}}
\end{gathered}
$$

where $B$ is the bulk modulus, $G$ is the average shear modulus and $\rho$ the density of the material. $\mathrm{P}$ and $\mathrm{S}$ refer to Primary and Secondary mode respectively. These velocities are the phase and group sound velocities the material would have if it were fully isotropic. Comparing with these is a consistent measure of how the material's acoustic properties deviate from isotropy, and allow for a direct comparison of anisotropy between different materials.

\section{Summary and outlook}

We have created a flexible and reliable piece of software for the calculation of phase and group sound velocities based on the stiffness tensor of a material. Power flow angles and enhancement factors are calculated accurately and 
efficiently. Sampling of wave vectors can be chosen freely, where denser grids are more computationally intensive, but provide a more detailed view of all quantities. The computational load scales linearly with the amount of grid points sampled, as every direction is fully independent of all others.

New features are still possible to add to christoffel. On performance, the most obvious first improvement would be parallelization support. Since each direction can be handled independently, several could be handled concurrently. When it comes to features to the christoffel module, there is detection of unstable strain modes and more elaborate options for calculating directional dependent shear and young moduli. For visualization, the first update will be to add support on projecting the data onto arbitrary unit cell shapes beyond cubic.

\section{Acknowledgements}

The authors would like to thank Michael Sluydts and Danny E.P. Vanpoucke for fruitful discussions and their assistance in hunting down elusive bugs. S.C. acknowledges financial support from OCAS NV by an OCASendowed chair at Ghent University.

\section{References}

[1] H. J. McSkimin, Ultrasonic measurement techniques applicable to small solid specimens, The Journal of the Acoustical Society of America 22 (4) (1950) 413-418. doi:10.1121/1.1906618.

[2] M. H. F. Sluiter, M. Weinert, Y. Kawazoe, Determination of the elastic tensor in low-symmetry structures, Europhysics Letters 43 (July) (1998) $183-188$.

[3] M. de Jong, W. Chen, T. Angsten, A. Jain, R. Notestine, A. Gamst, M. Sluiter, C. Krishna Ande, S. van der Zwaag, J. J. Plata, C. Toher, S. Curtarolo, G. Ceder, K. A. Persson, M. Asta, Charting the complete elastic properties of inorganic crystalline compounds, Scientific Data 2. doi:10.1038/sdata.2015.9.

[4] D. Muñoz-Santiburcio, A. Hernández-Laguna, J. I. Soto, AWESoMe: A code for the calculation of phase and group velocities of acoustic waves in homogeneous solids, Computer Physics Communications 192 (2015) 272-277. doi:10.1016/j.cpc.2015.02.024. 
[5] F. I. Fedorov, Theory of elastic waves in crystals, Springer, 1968.

[6] H. J. Maris, Enhancement of Heat Pulses in Crystals Due to Elastic Anisotropy, The Journal of the Acoustical Society of America 49 (1A) (1971) 88. doi:10.1121/1.1976094.

[7] G. A. Northrop, Acoustic phonon anisotropy: Phonon focusing, Computer Physics Communications 28 (1) (1982) 103-107. doi:10.1016/00104655(82)90065-0.

[8] K. B. Petersen, M. S. Pedersen, The matrix cookbook, version 20121115 (nov 2012). 

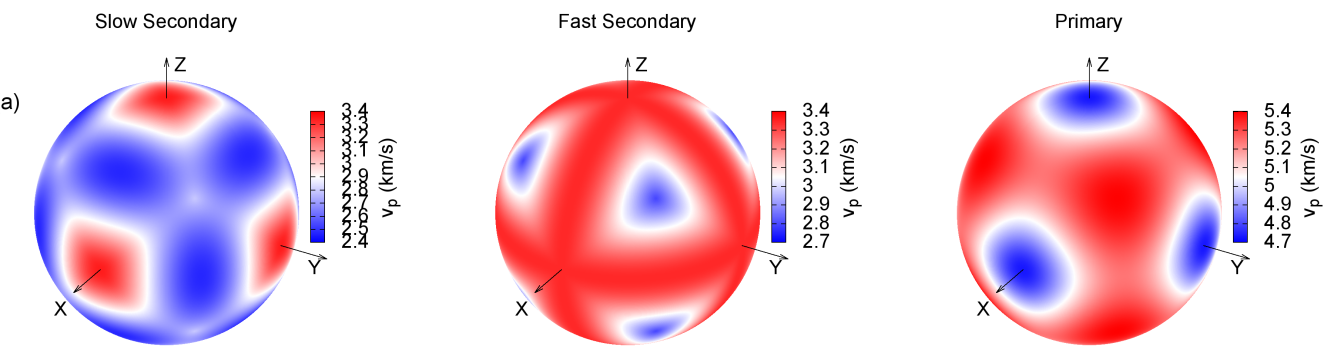

(b)
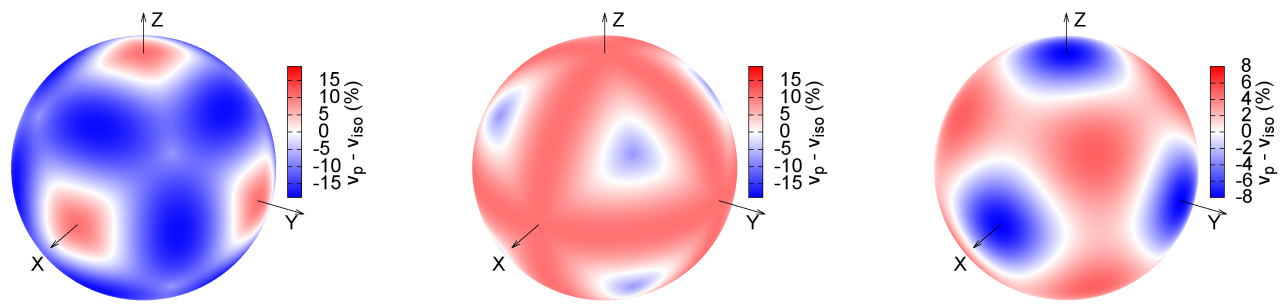

(c)
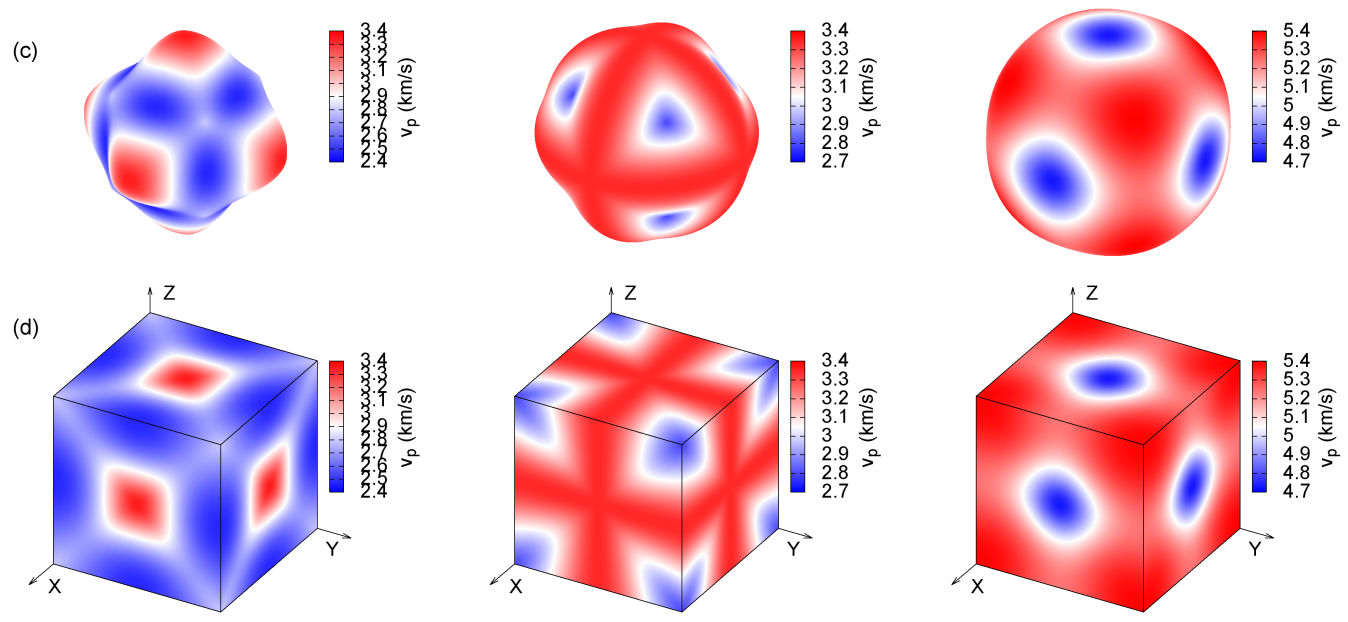

Figure 4: Phase velocities of GaAs projected in 3D. In the $\mathrm{X}, \mathrm{Y}$ and $\mathrm{Z}$ directions, the primary mode is slowest, while the secondary modes are fastest (though still slower than the primary). (a) The absolute velocities projected onto the unit sphere. (b) Same projection, though the colors now indicate velocity relative to the isotropic velocities as defined in (26). (c) The radius of the sphere is scaled according to the velocity, a clear visual indication of how the phase velocity changes with direction. It is clearly visible that the modes are ordered from slow (left) to fast (right). (d) Projection onto the unit cube. This is useful primarily for cubic materials, as there is a one-to-one correspondence to the conventional crystal unit cell. 
Slow Secondary

(a)

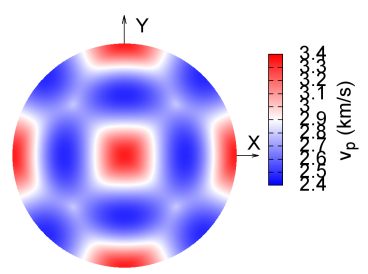

(b)

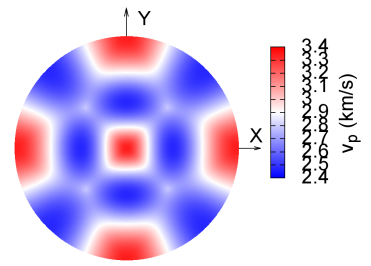

Fast Secondary
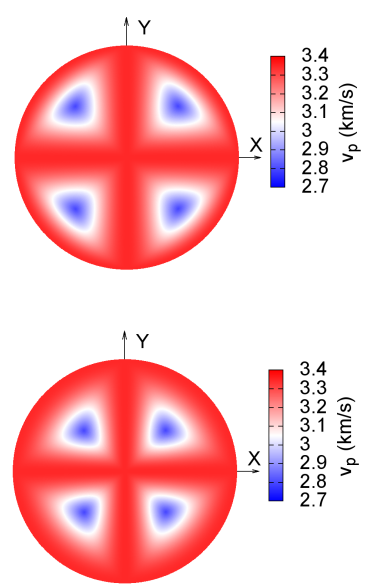

Primary
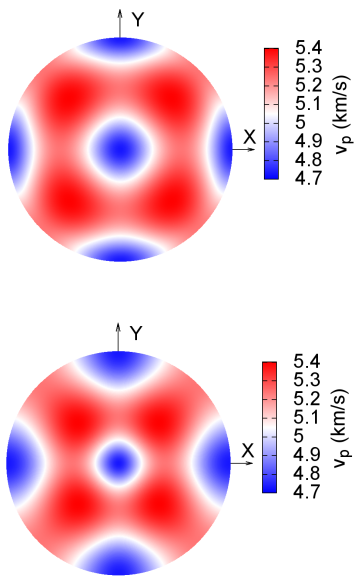

Figure 5: Phase velocities of GaAs projected onto the plane. In the X,Y and Z directions, the primary mode is slowest, while the secondary modes are fastest (though still slower than the primary). (a) Lambert azimuthal equal-area projection. Useful for comparing sound velocity behavior in different directions. It does not conserve angles. (b) Stereographic projection which conserves angles, thus locally preserves shape. Useful for studying local shapes or lines of interest.
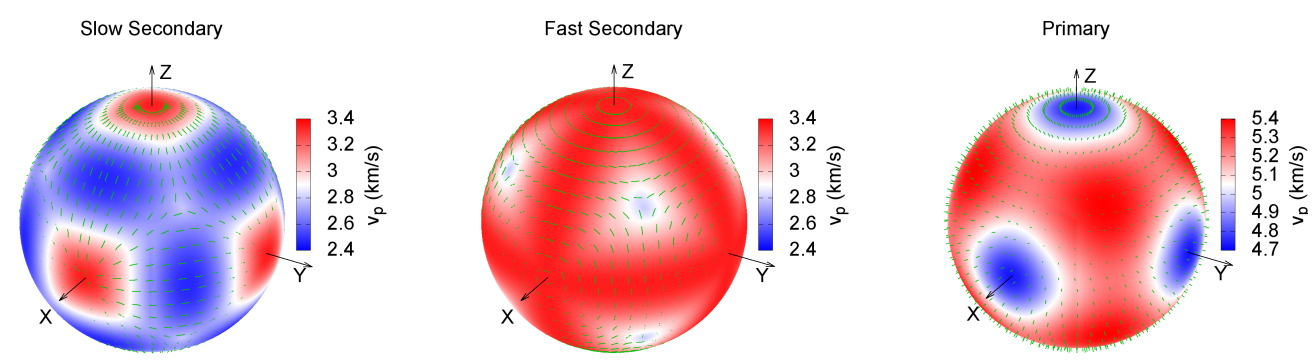

Figure 6: Polarization of sound waves in GaAs, superimposed over the phase velocities. It is clear that the primary mode is pseudo-longitudinaly polarized, while the secondary modes have a pseudo-transverse polarization. 
Slow Secondary

(a)

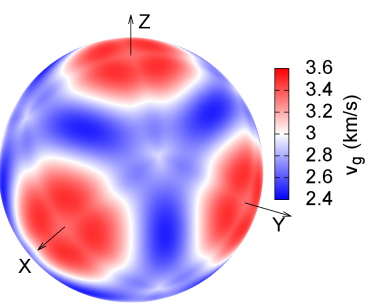

(b)

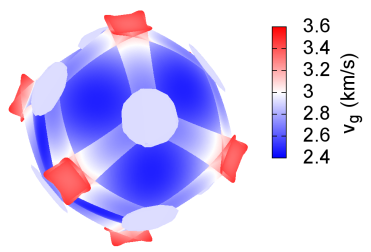

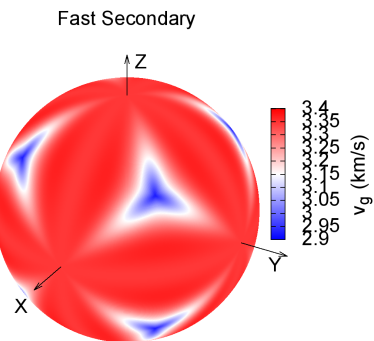
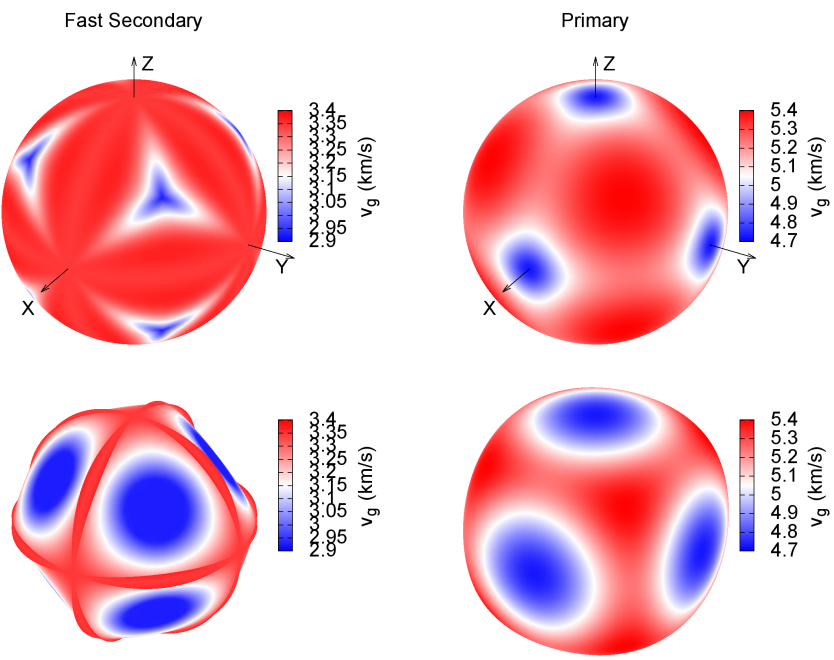

Figure 7: Group velocities of GaAs. (a) Projection of the group velocity corresponding to each phase direction on the unit sphere. (b) Full representation of the ray surfaces. The secondary modes show many caustics.
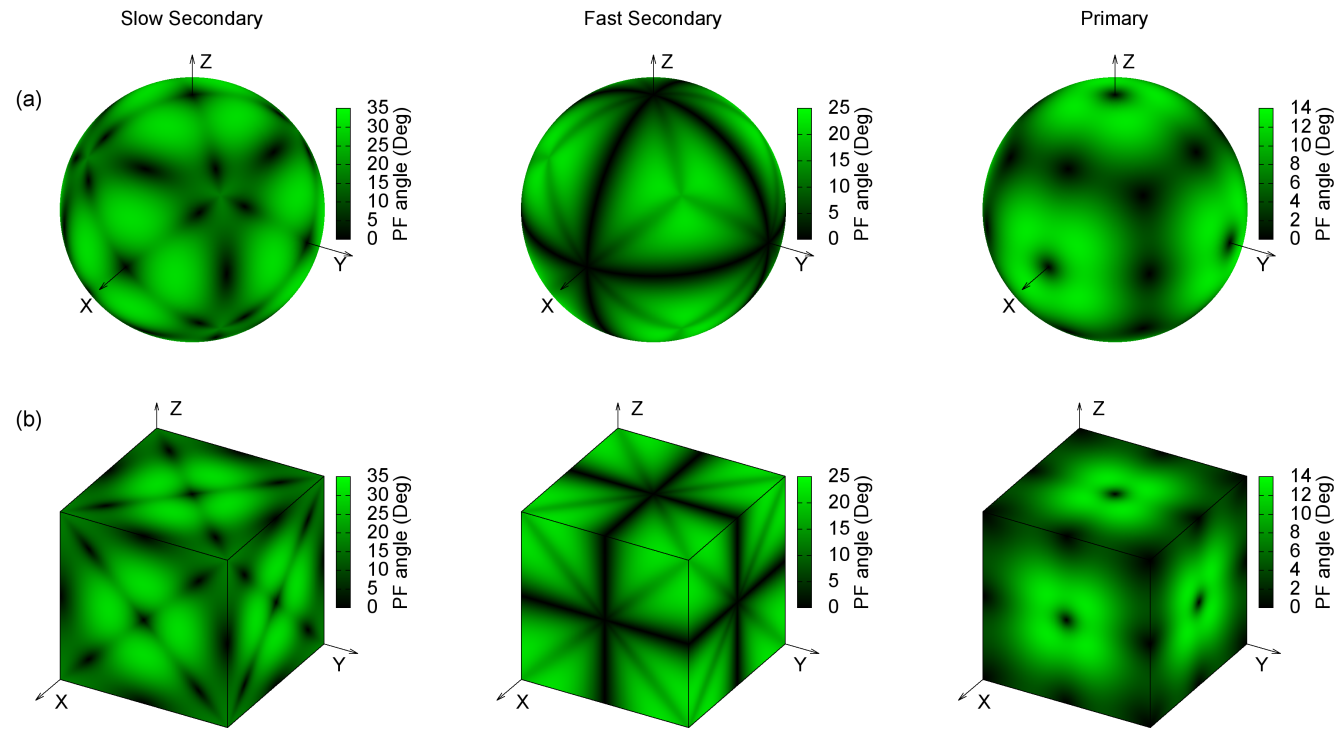

Figure 8: Power flow angles of GaAs as projected on the unit sphere (a) and unit cube (b). High symmetry points and lines of the fcc structure are clearly visible. 

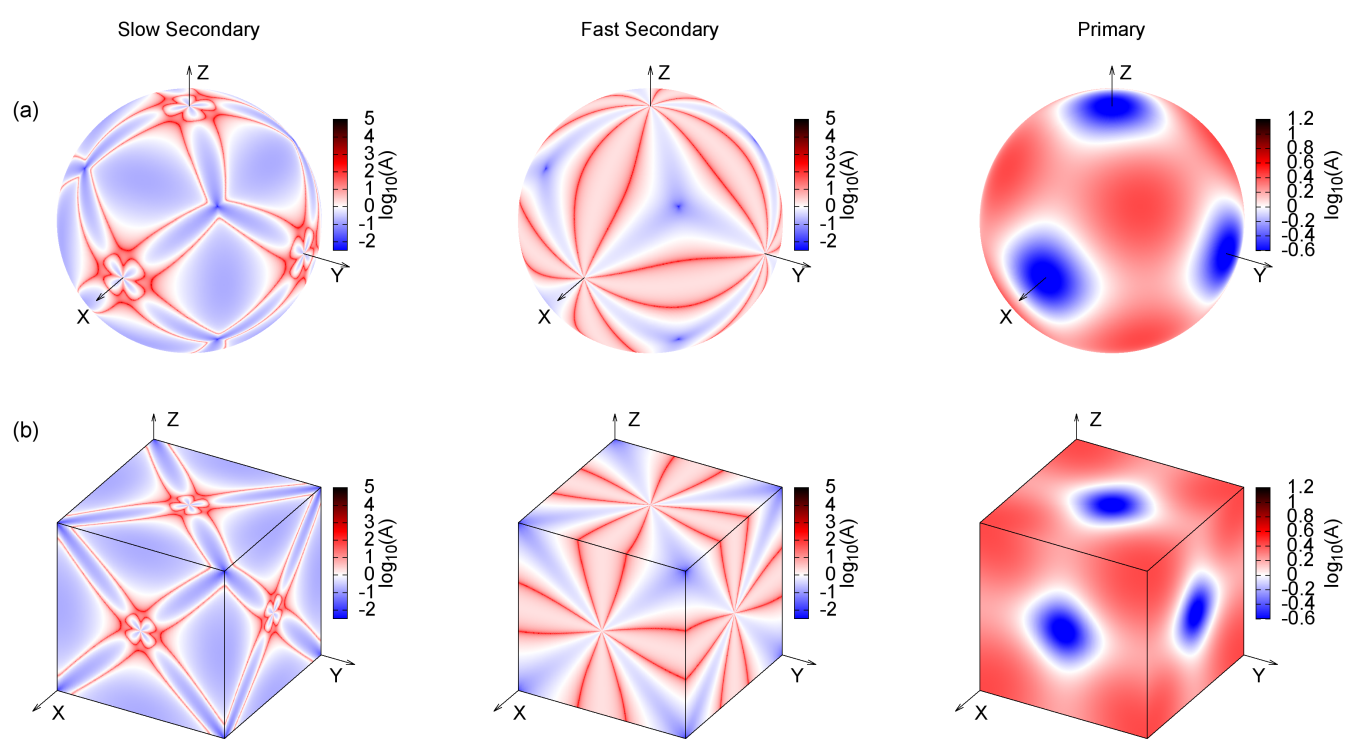

Figure 9: Enhancement factors of GaAs as projected on the unit sphere (a) and unit cube (b). The secondary modes exhibit a rich structure with narrow bands of extremely high enhacement. The primary mode shows more moderate enhancement, primarily along the cube vertices. 